\title{
Contribution of the AcrAB-TolC Efflux Pump to High-Level Fluoroquinolone Resistance in Escherichia coli Isolated from Dogs and Humans
}

\author{
Toyotaka SATO ${ }^{1)}$, Shin-ichi YOKOTA ${ }^{2)}$, Torahiko OKUBO ${ }^{1)}$, Kanako ISHIHARA ${ }^{1)}$, Hiroshi UENO ${ }^{3)}$, \\ Yasukazu MURAMATSU ${ }^{3)}$, Nobuhiro FUJII' ${ }^{2)}$ and Yutaka TAMURA ${ }^{1) *}$ \\ ${ }^{1)}$ Laboratory of Food Microbiology and Food Safety, Department of Health and Environmental Sciences, School of Veterinary Medicine, \\ Rakuno Gakuen University, 582 Midorimachi, Bunkyoudai, Ebetsu, Hokkaido 069-8501, Japan \\ 2) Department of Microbiology, Sapporo Medical University School of Medicine, South-1, West-17, Chuo-ku, Sapporo 060-8556, \\ Hokkaido, Japan \\ ${ }^{3)}$ Laboratory of Zoonoses, Department of Health and Environmental Sciences, School of Veterinary Medicine, Rakuno Gakuen University, \\ 582 Midorimachi, Bunkyoudai, Ebetsu, Hokkaido 069-8501, Japan
}

(Received 26 April 2012/Accepted 31 October 2012/Published online in J-STAGE 14 November 2012)

ABSTRACT. Fluoroquinolone resistance is mainly caused by mutations in quinolone resistance-determining regions of DNA gyrase and topoisomerase IV in Escherichia coli. The AcrAB-TolC efflux pump contributes to resistance against fluoroquinolone and other antimicrobials. In this study, we investigated a high-level mechanism of fluoroquinolone resistance in E. coli that was isolated from human clinical samples and canine fecal samples. E. coli strains with high levels of fluoroquinolone resistance have been found to be frequently resistant to cephalosporins. Strains with high-level fluoroquinolone resistance exhibited lower intracellular enrofloxacin (ENR) concentrations, higher expression of AcrA, and a greater reduction in the fluoroquinolone minimum inhibitory concentration for treatment with an efflux pump inhibitor. The frequency of strains with enhanced ENR resistance selection and the survival rate of $E$. coli in the presence of ENR in vitro were correlated well with AcrA protein expression levels in the parental strains. These results suggest that AcrAB-TolC efflux pump over-expression is related to high-level fluoroquinolone resistance and the selection of strains with enhanced fluoroquinolone resistance. KEY WORDS: $E$. coli, efflux pump, enrofloxacin, fluoroquinolone resistance.

doi: 10.1292/jvms.12-0186; J. Vet. Med. Sci. 75(4): 407-414, 2013

Antimicrobials that are intended for human use are also used on companion animals. Their excessive and inappropriate administration in animals leads to the generation and spread of antimicrobial-resistant bacteria, which in veterinary medicine represent a potential hazard to human health $[1,26,33,36]$. Companion animals live in close proximity to humans and receive medical treatment more frequently than food-producing animals [23]. Platell et al. reported that some fluoroquinolone-resistant Escherichia coli isolates that were derived from companion animals (i.e., dogs and cats) were very similar to the ones found in humans [27].

Fluoroquinolones are widely used to treat various bacterial infections in humans and companion animals. E. coli infections, especially urinary tract infections, are frequently treated with fluoroquinolones. Thus, fluoroquinolone resistance has increased among clinical isolates of humans and companion animals $[7,24]$. Previous studies reported that extended-spectrum $\beta$-lactamase (ESBL)-producing $E$. coli and Klebsiella pneumoniae are frequently resistant to fluoroquinolone $[20,30,32]$. Cephalosporins and fluoroquinolones

\footnotetext{
*Correspondence to: Tamura, Y., Laboratory of Food Microbiology and Food Safety, Department of Health and Environmental Sciences, School of Veterinary Medicine, Rakuno Gakuen University, 582 Midorimachi, Bunkyoudai, Ebetsu, Hokkaido 069-8501, Japan.

e-mail: tamuray@rakuno.ac.jp

(C)2013 The Japanese Society of Veterinary Science
}

are among the most important antimicrobials that are used on humans and animals, mainly because of their broad-spectrum effectiveness against both gram-positive and gram-negative bacteria. Therefore, the appearance of fluoroquinolone- and/ or cephalosporin-resistant bacteria is a serious problem in clinical medicine.

Fluoroquinolone resistance mechanisms are mainly based on chromosomal mutations in the quinolone resistancedetermining regions (QRDRs) of gyrA and gyrB, which encode DNA gyrase subunits, and parC and pare, which encode topoisomerase IV subunits [2, 8, 11, 37]. Resistance to nalidixic acid (NAL) and slightly decreased susceptibility to fluoroquinolones is attributed to a single mutation in $g y r A$. A secondary mutation in $g y r A$ and additional mutations in $\operatorname{par} C$ and/or $\operatorname{parE}$ are necessary for high-level fluoroquinolone resistance [8]. Moreover, plasmid-mediated quinolone resistance-mechanism determinants (PMQRs), such as the $q n r, a a c\left(6^{\prime}\right)-I b-c r$ and qepA genes, have been reported in gram-negative bacteria, including $E$. coli $[12,29,35]$. However, the acquisition of PMQRs results in only low-level fluoroquinolone resistance.

An alternative mechanism of fluoroquinolone resistance is decreased intracellular concentrations of antimicrobials. The over-expression of AcrAB-TolC, which is a major multidrug efflux pump in E. coli, and the down-regulation of the porin of the outer membrane protein $\mathrm{F}(\mathrm{OmpF})$ are caused by mutations or insertions in their regulatory genes [6]. AcrA is a component of AcrAB-TolC that belongs to the resistance-nodulation-division family of exporters, has 
a tripartite structure, and exhibits broad-substrate specificity [28]. Efflux pump- and/or porin-based resistance results in slightly decreased susceptibility to fluoroquinolone. These resistance mechanisms combined with QRDR mutations lead to high levels of fluoroquinolone-resistance [13]. In addition, efflux pump over-expression and a deficiency in porins also induce resistance to various other classes of antimicrobials, including cephalosporins [9]. Most of the effects of the AcrAB-TolC on multidrug resistance have been studied using laboratory strains, but not clinical isolates [13, 28]. In laboratory strains, AcrAB over-expression increases the MICs of several antimicrobials. However, in most of them, AcrAB over-expression alone does not modulate the phenotypic switch from susceptibility to resistance. Therefore, other resistance mechanisms are most likely to be responsible for the switch.

In this study, we investigated the mechanism of fluoroquinolone resistance by using human and canine $E$. coli isolates, and estimated the risk of transmitting fluoroquinoloneresistant $E$. coli from dogs to humans. We also examined the potential effect of AcrAB-TolC on the survival mechanism of $E$. coli in the presence of fluoroquinolone in vitro.

\section{MATERIALS AND METHODS}

Bacterial strains: E. coli strains were isolated from humans and dogs. Human strains were isolated and stocked at Sapporo Clinical Laboratories Inc. (Sapporo, Japan) and Hokkaido University Hospital (Sapporo, Japan) from 2008 to 2009. Human clinical samples were collected from the urine, pharynx, phlegm, feces, aspirate fluids, intestinal juice, wounds, vaginal fluid, pus and blood of outpatients and inpatients. Canine fecal samples were collected using cotton swabs from Rakuno Gakuen University Hospital (Ebetsu, Japan) and 8 animal clinics in Ebetsu City (Japan). The case histories of the dogs varied, including urinary tract infections, cystitis, chronic diarrhea, dermatitis and septicemia.

The $50 \mathrm{E}$. coli clinical isolates contained 12 enrofloxacin (ENR) susceptible strains [FQSECs; ENR minimum inhibitory concentration (MIC) ranged from 0.03 to $0.125 \mu \mathrm{g} / \mathrm{ml}$ ], 18 intermediate-level ENR-resistant strains (iFQRECs; ENR MIC ranged from 16 to $64 \mu \mathrm{g} / \mathrm{ml}$ ), and 20 high-level ENRresistant strains (hFQRECs; ENR MIC was more than 128 $\mu \mathrm{g} / \mathrm{ml})$. E. coli strain AG100, which was used as a reference strain for determining AcrA protein expression levels, was gifted by Dr. Helen I. Zgurskaya (University of Oklahoma, Norman, OK, U.S.A.).

Chemicals and media: Phe-Arg- $\beta$-naphthylamide (PA $\beta \mathrm{N})$ was purchased from Sigma-Aldrich (St. Louis, MO, U.S.A.), and 1-(1-naphthylmethyl)-piperazine (NMP) was obtained from Chess (Mannheim, Germany). Luria-Bertani (LB) broth and Mueller-Hinton (MH) agar were obtained from Oxoid (Basingsoke, U.K.). ENR and ciprofloxacin (CIP) were obtained from Bayer (Osaka, Japan), levofloxacin (LVX) was obtained from Daiichi-Sankyo (Tokyo, Japan), and NM394 [the active form of prulifloxacin (PUR)] was obtained from Meiji Seika Pharma (Tokyo, Japan). NAL was obtained from Wako Junyaku (Osaka, Japan). Cefazolin (CEZ), cefalexin
(CEX) and cefpodoxime (CPD) were obtained from SigmaAldrich.

Susceptibility testing: Susceptibility to a panel of antimicrobials was studied using the agar dilution method in the presence or absence of efflux pump inhibitor (EPI), PA $\beta \mathrm{N}$, and NMP, in accordance with the Clinical and Laboratory Standards Institute (CLSI) [4]. Staphylococcus aureus ATCC29213, Enterococcus faecalis ATCC29212, E. coli ATCC25922, and Pseudomonas aeruginosa ATCC27853 were used as quality control strains. CLSI breakpoints were used when available and antimicrobial breakpoints that were not included in the CLSI were defined by a population of the isolates that were tested in this study. The concentrations of $\mathrm{PA} \beta \mathrm{N}(80 \mu \mathrm{g} / \mathrm{m} l)$ and NMP $(160 \mu \mathrm{g} / \mathrm{m} l)$ were based on their available ranges, as previously described [14, 31].

$D N A$ amplification and sequencing of $Q R D R s$ and $P M Q R$ : Mutations in the QRDRs of the gyrA, parC, parE and gyrB genes were detected by direct DNA sequencing of their PCR products, as described in previous studies [27, 31]. Nucleotide sequences were determined using a BigDye Terminator v3.1 Cycle Sequencing Kit with a 3130 Genetic Analyzer (Applied Biosystems, Foster City, CA, U.S.A.). The PMQR genes $q n r A, q n r B, q n r S$, aac(6')-Ib-cr and qepA were detected as previously reported $[3,16,22]$.

Detection of $\beta$-lactamase genes: $\beta$-Lactamase genes were detected by PCR and direct DNA sequencing according to a method described in previous studies [17, 25, 38].

Accumulation assay: The intracellular ENR concentration was assayed using a fluorometric uptake assay [34] with ENR at a final concentration of $15 \mu \mathrm{g} / \mathrm{m} l$ and carbonyl cyanide m-chlorophenylhydrazone (CCCP, Sigma-Aldrich) at a final concentration of $150 \mu \mathrm{M}$. The data represent the mean value \pm standard errors (SE) that were calculated from 3 independent experiments.

Western blot analysis: Bacteria were cultured in LB broth until the logarithmic phase at $37^{\circ} \mathrm{C}$. Harvested cells were lysed with $1 \%$ sodium dodecyl sulfate (SDS) at $100^{\circ} \mathrm{C}$ for $10 \mathrm{~min}$. Protein concentrations were determined by the bicinchoninic acid assay (Thermo Scientific, Waltham, MA, U.S.A.) using horse immunoglobulin $\mathrm{G}$ as a standard. Protein $(1 \mu \mathrm{g})$ from the total cell lysate was used for SDS-polyacrylamide gel electrophoresis on a $12.5 \%$ polyacrylamide slab gel [18]. The proteins on the gel were transferred to an Immobilon-P polyvinylidene fluoride membrane (Millipore, Billerica, MA, U.S.A.), and the membrane was blocked with $5 \%(\mathrm{w} / \mathrm{v})$ skim milk in a buffer that contained $20 \mathrm{mM}$ Tris$\mathrm{HCl}(\mathrm{pH} 7.6), 137 \mathrm{mM} \mathrm{NaCl}$ and $0.1 \%$ Tween 20 (TBST) for $1 \mathrm{hr}$ with agitation. Then, the membrane was incubated with rabbit anti-AcrA polyclonal antibody (1:10,000 dilution) for $1 \mathrm{hr}$ at room temperature with agitation. The membrane was washed 3 times with TBST and incubated in an alkaline phosphatase-conjugated goat anti-rabbit immunoglobulin antibody (1:2,000 dilution; Biosource International, Camarillo, CA, U.S.A.). After washing with TBST, specific binding was visualized with tetrazolium bromochloroindolyl phosphate and nitro blue tetrazolium. The resulting bands were scanned with a flatbed scanner, and they were analyzed using the public domain ImageJ program (U. S. National Institutes of 
Health, Bethesda, MD, U.S.A.). This method was performed in duplicate.

Frequency of strains with enhanced fluoroquinolone resistance in vitro: Bacteria were cultured to the late logarithmic phase at $37^{\circ} \mathrm{C}$ in LB broth, harvested by centrifugation, and re-suspended in sodium phosphate buffer ( $\mathrm{pH}$ 7.2). Total colony-forming units (CFU) were measured by serial dilution on MH agar plates. The cells $\left(10^{9} \mathrm{CFU} / \mathrm{m} l\right)$ were inoculated on $\mathrm{MH}$ agar plates that contained ENR MIC of each isolate at 4 -fold concentration. The number of strains with enhanced fluoroquinolone resistance colonies was counted after $48 \mathrm{hr}$ of culturing. The frequency of strains with enhanced fluoroquinolone resistance was calculated, whereby the data represent the mean value \pm standard deviation (SD) of the results from 3 independent experiments.

Time-killing assay: Bacteria were cultured overnight at $37^{\circ} \mathrm{C}$ in $2 \mathrm{~m} l$ of LB broth, harvested, re-suspended in $2 \mathrm{~m} l$ of fresh LB broth, and incubated for $1.5 \mathrm{hr}$ at $37^{\circ} \mathrm{C}$ without agitating to reach the early stationary phase (approximate growth from $5 \times 10^{7}$ to $2 \times 10^{8} \mathrm{CFU} / \mathrm{m} l$ ). Then, 4-fold concentrated ENR MIC was added at 3, 6, and $9 \mathrm{hr}$. Following this, the number of CFU was determined at different time points by plating serial dilutions on $\mathrm{MH}$ agar plates, which were incubated for $18 \mathrm{hr}$ at $37^{\circ} \mathrm{C}$. This assay was performed for 3 independent experiments.

Statistical analysis: Statistical significance was determined by the Student's $t$-test and Fisher's exact test $(P<0.05)$.

\section{RESULTS}

Relationship between QRDR mutations and fluoroquinolone susceptibility: We investigated fluoroquinolone MICs and QRDR mutations (Tables 1 and 2). Similar to ENR, all the iFQRECs and hFQRECs were resistant to the other 3 fluoroquinolones (CIP, LVX, and PUR), and the FQSECs were susceptible to them. All the iFQRECs and hFQRECs were also resistant to NAL, with the MIC being greater than $128 \mu \mathrm{g} / \mathrm{ml}$ (data not shown).

All 38 iFQRECs and hFQRECs, with the exception of one strain, carried 3 or 4 mutations in gyrA (S83 and D87) and par $C$ (S80 and/or E84). A few strains that had these 3 mutations also carried a mutation in parC; A108T and in parE; E460A, and S458T, respectively. One strain had 2 mutations in the QRDR: S80I and E84V in parC (Table 1). For the strains that carried 2 or 3 mutations in the QRDR, the ENR MIC ranged from 16 to $64 \mu \mathrm{g} / \mathrm{m} l$, and for those that carried 4 mutations, the ENR MIC ranged from 32 to $>256 \mu \mathrm{g} / \mathrm{ml}$. Among the strains that shared the same mutations in the QRDRs, a 2- to $>4$-fold variation was observed for ENR MIC (Table 1). These phenomena were also observed for the other 3 fluoroquinolones.

Relationship between ENR resistance and CPD resistance: Sixteen isolates were resistant to CEZ, CEX, and CPD. Among them, 15 possessed $b l a_{\text {CTX-M-27 }}(\mathrm{n}=3), b l a_{\mathrm{CTX}-}$ $\mathrm{M}-14(\mathrm{n}=4)$, and bla $_{\mathrm{CMY}-2}(\mathrm{n}=8$; Table 1$)$. Another strain (CE4) had mutations in the chromosomal ampC promoter region (T-32A; data not shown).

The appearance of CPD resistance tended to be higher in the hFQRECs $(55.0 \%, 11$ of the 20 strains) than in the iFQRECs $(27.8 \%, 5$ of the 18 strains), and was significantly higher than that in the FQSECs $(0 \%$; Table 1$)$. Regarding CPD-susceptible and CPD-resistant classifications, high-level fluoroquinolone resistance was seen at a higher frequency in the CPD-resistant strains $(68.8 \%, 11$ of 16 strains) than in the CPD-susceptible isolates $(26.5 \%, 9$ of 34 strains; $P<0.05$, data not shown), whereas intermediate-level fluoroquinolone resistance was seen at a similar frequency in the CPD-resistant isolates $(31.3 \%, 5$ of 16 strains) and in the CPD-susceptible isolates (35.2\%, 12 of 34 strains).

Effects of efflux pump inhibitors on the fluoroquinolone MICs of clinical isolates: $\mathrm{PA} \beta \mathrm{N}(80 \mu \mathrm{g} / \mathrm{m} l)$ significantly influenced the fluoroquinolone MICs of the clinical isolates (2- to 64-fold reduction in ENR and CIP; 1- to 32-fold reduction in LVX; 1 - to 16-fold reduction in PUR; Table 1). In the fluoroquinolone-resistant $E$. coli isolates, the reductions in the MIC tended to increase with higher MIC for all the fluoroquinolones. The degree of ENR MIC reduction by $\mathrm{PA} \beta \mathrm{N}$ and NMP was significantly greater in the hFQREC strains than in the iFQREC and FQSEC strains (Fig. 1A-1 and $1 \mathrm{~A}-2)$. Other fluoroquinolone MICs were also reduced by 2 - to 32 -fold in the hFQRECs more than for the FQSEC and $\mathrm{iFQREC}$ strains with lower MICs. In the FQSECs, the reductions in fluoroquinolone MICs were greater in the strains that had higher fluoroquinolone MICs (Table 2).

Determination of intracellular ENR concentrations: The intracellular ENR concentrations were significantly lower $(P<0.05)$ in the hFQRECs compared with the FQSECs, and they tended to be lower compared with the iFQRECs (Fig. $1 C-1)$. In the presence of CCCP, all the hFQRECs showed a significant increase in their intracellular ENR concentration compared with the FQSECs. However, there were no differences in the iFQRECs (Fig. 1C-2). We calculated the excretion of ENR by subtracting the intracellular ENR concentration in the absence of CCCP from that in the presence of CCCP (Fig. 1C-3). The hFQRECs and iFQRECs showed significantly higher efflux activity than the FQSECs, and the efflux activity of the hFQRECs tended to be higher than that of the iFQRECs.

Analysis of AcrA expression: AcrA expression in the hFQRECs was significantly higher than that in the iFQRECs and FQSECs (Table 1 and Fig. 1B). The ENR MIC was correlated with the AcrA expression level for each QRDR genotype (Table 1). AcrA expression in the iFQRECs showed no significant difference from that in the FQSECs (Fig. 1B).

HUE6, which is a human hFQREC strain, showed lower AcrA expression, despite the low ENR concentration in the absence of CCCP compared with other isolates with the same QRDR genotype (Table 1). In contrast, RE63, which is an iFQREC that was isolated from dogs, showed higher AcrA expression compared with other isolates with the same ENR MIC and QRDR genotypes.

Frequency of strains with enhanced fluoroquinolone resistance in vitro: The frequency of strains with enhanced fluoroquinolone resistance was determined by in vitro exposure to ENR by using the FQSECs (Table 2). The frequency of strains with enhanced fluoroquinolone resistance among the 


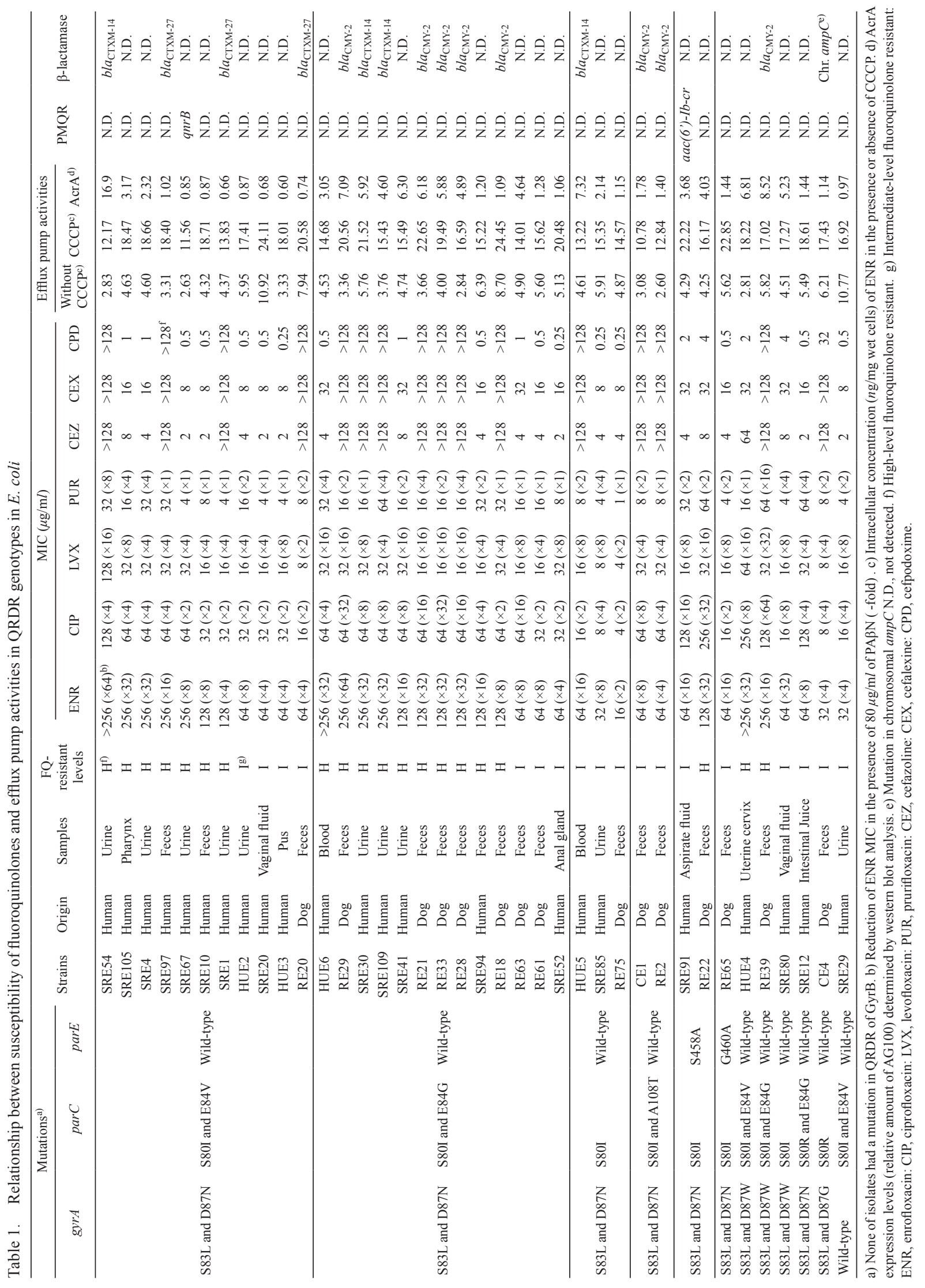


(A-1)

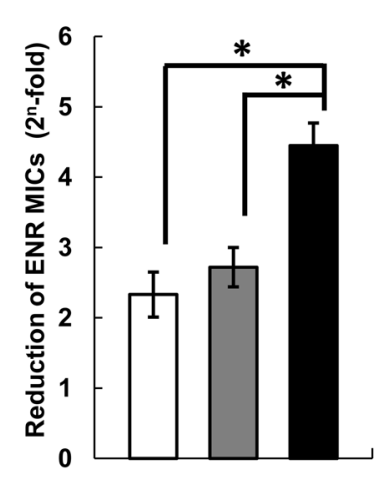

(C-1)

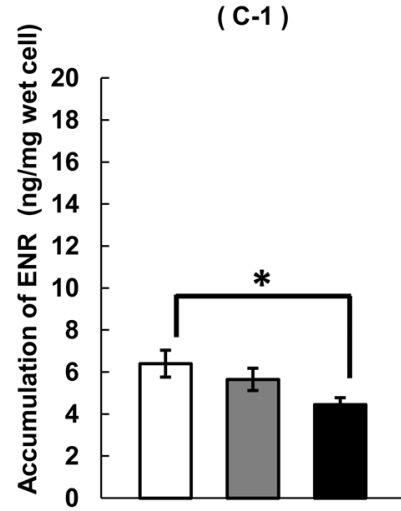

(A-2)

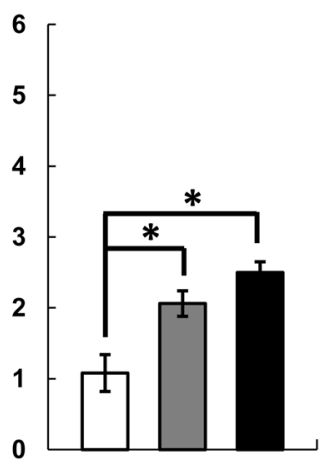

(C-2)

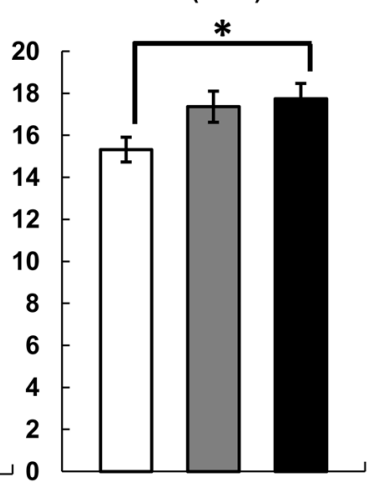

(B)

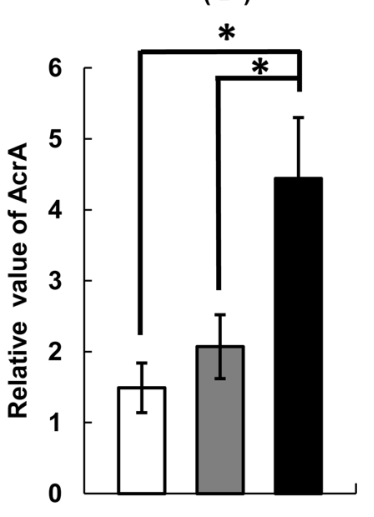

(C-3)

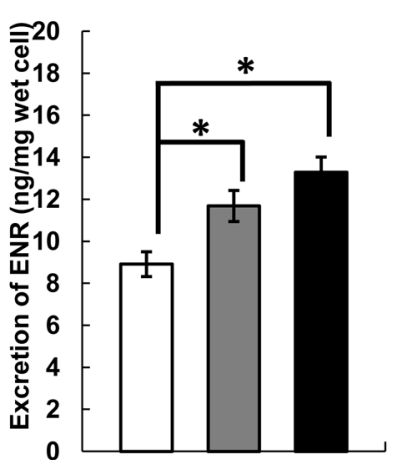

Fig. 1. The effect of the efflux pump at different ENR MICs $(\mu \mathrm{g} / \mathrm{m} l)$ in $E$. coli clinical isolates. A total of 50 isolates were used in these 3 experiments: 12 were FQSECs, 18 were iFQRECs and 20 were hFQRECs. A, the effects of PA $\beta \mathrm{N}(80 \mu \mathrm{g} / \mathrm{m} l, \mathrm{~A}-1)$ and NMP $(160 \mu \mathrm{g} / \mathrm{ml}, \mathrm{A}-2)$. B, Western blot analysis of AcrA. The AcrA expression level is shown as the relative value compared to that obtained for strain AG100 (wild-type expression of AcrA) that was used to assess the relative volume of AcrA above the wild-type levels. C, Intracellular concentration $(\mathrm{ng} / \mathrm{mg}$ wet cells) of ENR in the absence of CCCP $(\mathrm{C}-1)$ and in the presence of $\mathrm{CCCP}(\mathrm{C}-2)$. The excretion of ENR was calculated by subtracting the intracellular ENR concentration from that in the presence of CCCP (C-3; $n \mathrm{~g} / \mathrm{mg}$ wet cells). The data represent the mean value $\pm \mathrm{SE} . * P<0.05$.

strains with low AcrA expression (relative values to strain AG100; less than unity) ranged from $0.2 \times 10^{-9}$ to $3.7 \times 10^{-9}$, and those in strains with moderate AcrA expression (relative values to strain AG100; more than unity) ranged from $1.5 \times$ $10^{-8}$ to $5.8 \times 10^{-8}$. The frequency of selection of strains with enhanced ENR resistance was significantly correlated with the level of AcrA expression $\left(\mathrm{r}^{2}=0.41, P<0.05\right)$, whereas the frequency was not correlated with the QRDR mutations in gyrA and gyrB (Table 2).

Time-killing assay: We investigated the survival rate for susceptible isolates that were exposed to ENR and CPD (Table 2). The survival rate for those that were exposed to ENR at $6 \mathrm{hr}$ was significantly correlated with the frequency of strains with enhanced ENR resistance $\left(\mathrm{r}^{2}=0.67, P<0.01\right)$ and the level of AcrA expression $\left(r^{2}=0.66, P<0.01\right)$. The survival rate for those that were exposed to CPD at $9 \mathrm{hr}$ was also correlated with the level of AcrA expression $\left(\mathrm{r}^{2}=0.52\right.$, $P<0.05)$.

\section{DISCUSSION}

This study has revealed the mechanism of fluoroquinolone resistance in $E$. coli that was isolated from humans and dogs, and the contribution of AcrAB-TolC to its survival in the presence of fluoroquinolone in vitro. We found no apparent differences in the fluoroquinolone-resistance mechanisms when we compared $E$. coli that was isolated from dogs and humans. Further work is necessary to clarify the risk of transmitting fluoroquinolone-resistant $E$. coli from dogs to humans.

The degree of fluoroquinolone resistance in E. coli that was isolated from dogs and humans was primarily determined by the number of QRDR mutations that each bacterium had (Table 1). However, the hFQRECs had a wider range of ENR MIC than the iFQRECs regardless of the same QRDR genotypes, which could not be explained solely by the QRDR mutations. EPIs dramatically reduced fluoroquinolone MICs for almost all the clinical isolates, and the intracellular ENR concentration increased in the presence of CCCP (Table 1 
Table 2. Appearance frequency of strains with enhanced ENR-resistance, expression levels of AcrA protein and survival rate in ENR containing media of FQSECs

\begin{tabular}{|c|c|c|c|c|c|c|c|c|c|c|c|c|}
\hline \multirow{2}{*}{ Strains } & \multicolumn{7}{|c|}{$\mathrm{MIC}(\mu \mathrm{g} / \mathrm{m} l)$} & \multirow{2}{*}{$\begin{array}{c}\text { AcrA } \\
\text { expression } \\
\left.\text { level }(s)^{b}\right)\end{array}$} & \multirow{2}{*}{$\begin{array}{c}\text { Appearance } \\
\text { frequency of } \\
\text { ENR-resistant } \\
\text { mutant }^{c)}\end{array}$} & \multirow{2}{*}{$\begin{array}{c}\text { QRDRs } \\
\text { mutation of } \\
\text { ENR-resistant } \\
\text { mutant }\end{array}$} & \multirow{2}{*}{$\begin{array}{c}\text { ENR MIC of } \\
\text { ENR-resistant } \\
\text { mutant } \\
(\mu \mathrm{g} / \mathrm{m} l)\end{array}$} & \multirow{2}{*}{$\begin{array}{c}\text { Survival rate } \\
\text { of ENR for } \\
6 \mathrm{hr} \\
\left(\times 10^{-2}\right)^{\mathrm{d})}\end{array}$} \\
\hline & ENR & CIP & LVX & PUR & CEZ & CEX & CPD & & & & & \\
\hline RE15 & $0.03\left(\times 2^{\mathrm{a})}\right)$ & $0.01(\times 2)$ & $0.01(\times 2)$ & $0.03(\times 1)$ & 1 & 4 & 0.25 & 0.18 & $1.4 \pm 0.6$ & S83L(GyrA) & 0.25 & 5.4 \\
\hline CE52 & $0.03(\times 2)$ & $0.01(\times 2)$ & $0.03(\times 4)$ & $0.03(\times 1)$ & 2 & 8 & 0.5 & 0.61 & $3.5 \pm 1.7$ & S83L(GyrA) & 0.25 & 6.4 \\
\hline CE51 & $0.03(\times 4)$ & $0.03(\times 4)$ & $0.03(\times 4)$ & $0.03(\times 1)$ & 2 & 8 & 0.5 & 0.79 & $0.2 \pm 0.04$ & D87G(GyrA) & 0.25 & 1.1 \\
\hline CE55 & $0.03(\times 4)$ & $0.03(\times 2)$ & $0.03(\times 4)$ & $0.03(\times 1)$ & 2 & 8 & 0.5 & 0.87 & $3.7 \pm 3.1$ & S83L(GyrA) & 0.5 & 31.3 \\
\hline RE6 & $0.03(\times 4)$ & $0.03(\times 2)$ & $0.06(\times 4)$ & $0.03(\times 1)$ & 1 & 8 & 0.5 & 1.22 & $12.7 \pm 6.2$ & S83L(GyrA) & 0.5 & 32.5 \\
\hline RE13 & $0.03(\times 4)$ & $0.03(\times 2)$ & $0.06(\times 4)$ & $0.03(\times 2)$ & 1 & 8 & 0.5 & 1.26 & $10.9 \pm 3.8$ & S83L(GyrA) & 0.5 & 22.2 \\
\hline RE10 & $0.03(\times 4)$ & $0.03(\times 2)$ & $0.03(\times 4)$ & $0.03(\times 2)$ & 2 & 8 & 0.5 & 1.29 & $15.0 \pm 3.5$ & D87Y(GyrA) & 0.25 & 17.4 \\
\hline CE58 & $0.03(\times 4)$ & $0.03(\times 4)$ & $0.06(\times 8)$ & $0.03(\times 1)$ & 2 & 8 & 0.5 & 1.32 & $24.0 \pm 3.0$ & S83L(GyrA) & 0.5 & 24.7 \\
\hline RE14 & $0.03(\times 4)$ & $0.03(\times 4)$ & $0.06(\times 8)$ & $0.03(\times 2)$ & 2 & 16 & 0.5 & 1.59 & $25.8 \pm 8.8$ & D87Y(GyrA) & 0.25 & 28.2 \\
\hline RE12 & $0.06(\times 8)$ & $0.03(\times 4)$ & $0.06(\times 4)$ & $0.03(\times 1)$ & 2 & 8 & 0.5 & 1.79 & $25.0 \pm 4.7$ & S83L(GyrA) & 0.5 & 54.4 \\
\hline CE54 & $0.06(\times 8)$ & $0.06(\times 8)$ & $0.125(\times 8)$ & $0.06(\times 2)$ & 2 & 16 & 1 & 1.85 & $40.0 \pm 19.3$ & D426N(GyrB) & 0.25 & 52.3 \\
\hline RE1 & $0.125(\times 64)$ & $0.125(\times 16)$ & $0.125(\times 16)$ & $0.125(\times 4)$ & 4 & 32 & 2 & 4.98 & $58.8 \pm 9.5$ & S83L(GyrA) & 1 & 54.9 \\
\hline
\end{tabular}

a) Reduction of ENR MIC in the presence of $80 \mu \mathrm{g} / \mathrm{m} l$ of PABN (-fold). b) AcrA expression levels (relative amount of AG100) determined by western blot analysis. c) Emerging frequency of strains with enhanced ENR resistance $\left(\times 10^{-9}\right)$. Data represent the mean value \pm standard deviation of the results from 3 independent experiments. d) Time-killing assay following exposure to ENR for $6 \mathrm{hr}$. The value represents the relative number of CFU at $0 \mathrm{hr}$. ENR, enrofloxacin: CIP, ciprofloxacin: LVX, levofloxacin: PUR, prurifloxacin: CEZ, cefazoline: CEX, cefalexine: CPD, cefpodoxime.

and Fig. 1). The influence of fluoroquinolone MIC by EPIs and AcrA expression was clearly higher in the hFQRECs than in the iFQRECs and FQSECs. These data suggest that efflux pump over-expression in combination with QRDR mutations leads to extremely high-level fluoroquinolone resistance. These results are consistent with the previously described in E. coli and Salmonella enterica serovar Typhimurium [9, 10]. However, high-level fluoroquinolone resistance could not be completely explained by the QRDR mutations and AcrAB-TolC expression in the clinical isolates.

Efflux pump over-expression should enhance the frequency of strains with enhanced fluoroquinolone resistance, because it decreases the intracellular fluoroquinolone concentration. Consistent with this, the frequency of strains with enhanced fluoroquinolone resistance was higher for susceptible isolates of Salmonella enterica serovar Choleraesuis with low intracellular ENR concentrations [34]. In this study, strains that exhibited higher AcrA expression showed higher ENR MICs, and we observed a correlation between the levels of AcrA expression, survival in ENR, and the frequency of QRDR mutations (Table 2). In addition, among the strains that had enhanced ENR resistance and contained the same QRDR mutation, the FQSECs with moderate AcrA expression had higher fluoroquinolone MICs compared with the FQSECs with low AcrA expression (Table 2). These results suggest that the over-expression of AcrABTolC decreases the intracellular ENR concentration largely compared to the low expression of AcrAB-TolC, and thus provides a strong absolute survival advantage in the presence of fluoroquinolone. Although we could not explain this relationship directly, this advantage can be used to select for strains with enhanced ENR resistance and make the first-step QRDR mutation easier to occur in FQSECs with moderate AcrA expression than FQSECs with low AcrA expression. Therefore, AcrAB-TolC efflux pump over-expression may not be limited to generating high-level fluoroquinolone re- sistance, but it may be related to the appearance of first-step fluoroquinolone-resistant mutants.

In addition, the hFQREC strains were frequently CPD-resistant (Table 1). A suggested mechanism for the appearance of fluoroquinolone and cephalosporin concomitant resistance is the co-localization of ESBL and PMQR determinants in the same genetic platform $[15,19]$. However, in this study, we did not find any strains that harbored such co-localizations, which suggests that there may be other concomitant fluoroquinolone and cephalosporin-resistance mechanisms.

Efflux pump over-expression is responsible for resistance to fluoroquinolones and other antimicrobials [28]. Our data show that over-expression of the efflux pump that is mediated by AcrA is associated with the multidrug-resistant phenotype (cephalosporins, CHL, NAL and fluoroquinolones) in E. coli clinical isolates (data not shown). In CPD-susceptible strains, CPD MIC was higher with higher AcrA expression (Tables 1 and 2). Previous studies showed that a decrease in OmpF expression enhances cephalosporin and fluoroquinolone MICs [6, 21], and $\mathrm{OmpF}$ has been shown to be regulated by MarA, which also regulates AcrAB expression [5, 6, 13]. These facts also indicate that AcrAB-TolC may influence cephalosporin susceptibility in clinical isolates. More number of definitive studies is needed to elucidate the relationship between AcrAB-TolC and cephalosporin resistance.

In conclusion, high levels of fluoroquinolone resistance are caused by the upregulation of efflux mechanisms in addition to QRDR mutations in both human and canine E. coli isolates. In addition, AcrAB-TolC over-expression confers QRDR mutations and survival advantages with respect to ENR exposure.

ACKNOWLEDGMENTS. We thank Dr. Helen I. Zgurskaya, University of Oklahoma, for providing anti-AcrA antibody and E. coli strain AG100. We also thank Mr. Osamu Kuwahara, Sapporo Clinical Laboratory, Inc., and Mr. Hirotsugu 
Akizawa, Clinical Laboratory, Hokkaido University Hospital, for providing us E. coli clinical isolates. Finally, we thank Dr. Masaru Usui, National Veterinary Assay Laboratory, for his excellent technical instruction on how to perform the fluoroquinolone excretion assay. Funding for this research came in the form of a grant-in-aid from the Japanese Ministry of Health, Labour, and Welfare (H21-Shokuhin-Ippan-013), and a grant program for developing the supporting system for upgrading education and research from the Japan Ministry of Education, Culture, Sports, Science and Technology.

\section{REFERENCES}

1. Aarestrup, F. M. 1999. Association between the consumption of antimicrobial agents in animal husbandry and the occurrence of resistant bacteria among food animals. Int. J. Antimicrob. Agents 12: 279-285. [Medline] [CrossRef]

2. Breines, D. M., Ouabdesselam, S., Ng, E. Y., Tankovic, J., Shah, S., Soussy, C. J. and Hooper, D. C. 1997. Quinolone resistance locus $n f x D$ of Escherichia coli is a mutant allele of the parE gene encoding a subunit of topoisomerase IV. Antimicrob. Agents Chemother. 41: 175-179. [Medline]

3. Cattoir, V., Poirel, L., Rotimi, V., Soussy, C. J. and Nordmann, P. 2007. Multiplex PCR for detection of plasmid-mediated quinolone resistance qnr genes in ESBL-producing enterobacterial isolates. J. Antimicrob. Chemother. 60: 394-397. [Medline] [CrossRef]

4. Clinical and Laboratory Standards Institute. 2002. Performance standards for antimicrobial disk and dilution susceptibility tests for bacteria isolated from animals. Approved standard, 2nd ed. NCCLS document M31-A2. Clinical and Laboratory Standards Institute, Wayne.

5. Cohen, S. P., McMurry, L. M., Hooper, D. C., Wolfson, J. S. and Levy, S. B. 1989. Cross-resistance to fluoroquinolones in multiple-antibiotic-resistant (Mar) Escherichia coli selected by tetracycline or chloramphenicol: decreased drug accumulation associated with membrane changes in addition to OmpF reduction. Antimicrob. Agents Chemother. 33: 1318-1325. [Medline] [CrossRef]

6. Cohen, S. P., McMurry, L. M. and Levy, S. B. 1988. marA locus causes decreased expression of $\mathrm{OmpF}$ porin in multiple-antibiotic-resistant (Mar) mutants of Escherichia coli. J. Bacteriol. 170: 5416-5422. [Medline]

7. Cohn, L. A., Gary, A. T., Fales, W. H. and Madsen, R. W. 2003. Trends in fluoroquinolone resistance of bacteria isolated from canine urinary tracts. J. Vet. Diagn. Invest. 15: 338-343. [Medline] [CrossRef]

8. Conrad, S., Oethinger, M., Kaifel, K., Klots, G., Marre, R. and Kern, W. V. 1996. gyrA mutations in high-level fluoroquinoloneresistant clinical isolates of Escherichia coli. J. Antimicrob. Chemother. 38: 443-455. [Medline] [CrossRef]

9. Eaves, D. J., Ricci, V. and Piddock, L. J. 2004. Expression of acrB, acrF, acrD, marA, and soxS in Salmonella enterica serovar Typhimurium: role in multiple antibiotic resistance. Antimicrob. Agents Chemother. 48: 1145-1150. [Medline] [CrossRef]

10. Everett, M. J., Jin, Y. F., Ricci, V. and Piddock, L. J. 1996. Contributions of individual mechanisms to fluoroquinolone resistance in 36 Escherichia coli strains isolated from humans and animals. Antimicrob. Agents Chemother. 40: 2380-2386. [Medline]

11. Heisig, P. 1996. Genetic evidence for a role of $\operatorname{parC}$ mutations in development of high-level fluoroquinolone resistance in
Escherichia coli. Antimicrob. Agents Chemother. 40: 879-885. [Medline]

12. Jacoby, G. A. 2005. Mechanisms of resistance to quinolones. Clin. Infect. Dis. 41: S120-S126. [Medline] [CrossRef]

13. Jellen-Ritter, A. S. and Kern, W. V. 2001. Enhanced expression of the multidrug efflux pumps AcrAB and AcrEF associated with insertion element transposition in Escherichia coli mutants selected with a fluoroquinolone. Antimicrob. Agents Chemother. 45: 1467-1472. [Medline] [CrossRef]

14. Kern, W. V., Steinke, P., Schumacher, A., Schuster, S., Von, B. H. and Bohnert, J. A. 2006. Effect of 1-(1-naphthylmethyl)piperazine, a novel putative efflux pump inhibitor, on antimicrobial drug susceptibility in clinical isolates of Escherichia coli. J. Antimicrob. Chemother. 57: 339-343. [Medline] [CrossRef]

15. Kim, E. S., Jeong, J. Y., Jun, J. B., Choi, S. H., Lee, S. O., Kim, M. N., Woo, J. H. and Kim, Y. S. 2009. Prevalence of aac(6') $\mathrm{Ib}$-cr encoding a ciprofloxacin-modifying enzyme among Enterobacteriaceae blood isolates in Korea. Antimicrob. Agents Chemother. 53: 2643-2645. [Medline] [CrossRef]

16. Kim, H. B., Park, C. H., Kim, C. J., Kim, E. C., Jacoby, G. A. and Hooper, D. C. 2009. Prevalence of plasmid-mediated quinolone resistance determinants over a 9-year period. Antimicrob. Agents Chemother. 53: 639-645. [Medline] [CrossRef]

17. Kojima, A., Ishii, Y., Ishihara, K., Esaki, H., Asai, T., Oda, C., Tamura, Y., Takahashi, T. and Yamaguchi, K. 2005. Extendedspectrum-beta-lactamase-producing Escherichia coli strains isolated from farm animals from 1999 to 2002: report from the Japanese Veterinary Antimicrobial Resistance Monitoring Program. Antimicrob. Agents Chemother. 49: 3533-3537. [Medline] [CrossRef]

18. Laemmli, U. K. 1970. Cleavage of structural proteins during the assembly of the head of bacteriophage T4. Nature 227: 680-685. [Medline] [CrossRef]

19. Lavigne, J. P., Marchandin, H., Delamas, J., Bouziges, N., Lecaillon, E., Cavalie, L., Jean-Pierre, H., Bonnet, R. and Sotto, A. 2006. qnrA in CTX-M-producing Escherichia coli isolates from France. Antimicrob. Agents Chemother. 50: 4224-4228. [Medline] [CrossRef]

20. Martínez-Martínez, L., Pascual, A., Conejo Mdel, C., García, I., Joyanes, P., Doménech-Sánchez, A. and Benedí, V. J. 2002. Energy-dependent accumulation of norfloxacin and porin expression in clinical isolates of Klebsiella pneumoniae and relationship to extended-spectrum beta-lactamase production. Antimicrob. Agents Chemother. 46: 3926-3932. [Medline] [CrossRef]

21. Mortimer, P. G. and Piddock, L. J. 1993. The accumulation of five antimicrobial agents in porin-deficient mutations of Escherichia coli. J. Antimicrob. Chemother. 32: 195-213. [Medline] [CrossRef]

22. Park, C. H., Robicsek, A., Jacoby, G. A., Sahm, D. and Hooper, D. C. 2006. Prevalence in the United States of $a a c\left(6^{\prime}\right)-I b-c r$ encoding a ciprofloxacin-modifying enzyme. Antimicrob. Agents Chemother. 50: 3953-3955. [Medline] [CrossRef]

23. Pedersen, K., Pedersen, K., Jensen, H., Finster, K., Jensen, V. F. and Heuer, O. E. 2007. Occurrence of antimicrobial resistance in bacteria from diagnostic samples from dogs. J. Antimicrob. Chemother. 60: 775-781. [Medline] [CrossRef]

24. Peña, C., Albareda, J. M., Pallares, R., Pujol, M., Tubau, F. and Ariza, J. 1995. Relationship between quinolone use and appearance of ciprofloxacin-resistant Escherichia coli in bloodstream infections. Antimicrob. Agents Chemother. 39: 520-524. [Medline] [CrossRef]

25. Pérez-Pérez, F. J. and Hanson, N. D. 2002. Detection of plasmid- 
mediated AmpC beta-lactamase genes in clinical isolates by using multiplex PCR. J. Clin. Microbiol. 40: 2153-2162. [Medline] [CrossRef]

26. Phillips, I., Casewell, M., Cox, T., De Groot, B., Friis, C., Jones, R., Nightingale, C., Preston, R. and Waddell, J. 2004. Dose the use of antibiotics in food animals pose a risk to human health? A critical review of published data. J. Antimicrob. Chemother. 53: 28-52. [Medline] [CrossRef]

27. Platell, J. L., Cobbold, R. N., Johnson, J. R., Heisig, A., Heisig, P., Clabots, C., Kuskowski, M. A. and Trott, D. J. 2011. Commonality among fluoroquinolone-resistant sequence type ST131 extraintestinal Escherichia coli isolates from humans and companion animals in Australia. Antimicrob. Agents Chemother. 55: 3782-3787. [Medline] [CrossRef]

28. Poole, K. 2005. Efflux-mediated antimicrobial resistance. $J$. Antimicrob. Chemother. 56: 20-51. [Medline] [CrossRef]

29. Robicsek, A., Strahilevitzl, J., Jacoby, G. A., Macielag, M., Abbanat, D., Park, C. H., Bush, K. and Hooper, D. C. 2006. Fluoroquinolone-modifying enzyme: a new adaptation of a common aminoglycoside acetyltransferase. Nat. Med. 12: 83-88. [Medline] [CrossRef]

30. Ruppé, E., Hem, S., Lath, S., Gautier, V., Ariey, F., Sarthou, J. L., Monchy, D. and Arlet, G. 2009. CTX-M beta-lactamases in Escherichia coli from community-acquired urinary tract infections, Cambodia. Emerg. Infect. Dis. 15: 741-748. [Medline] [CrossRef]

31. Sáenz, Y., Ruiz, J., Zarazaga, M., Teixidó, M., Torres, C. and Vila, J. 2004. Effect of the efflux pump inhibitor Phe-Arg-betanaphthylamide on the MIC values of the quinolones, tetracycline and chloramphenicol, in Escherichia coli isolates of different origin. J. Antimicrob. Chemother. 53: 544-545. [Medline]
[CrossRef]

32. Sorlozano, A., Gutierrez, J., Jimenez, A., de Dios Luna, J. and Martinez, J. L. 2007. Contribution of a new mutation in parE to quinolone resistance in extended-spectrum-beta-lactamaseproducing Escherichia coli isolates. J. Clin. Microbiol. 45: 2740-2742. [Medline] [CrossRef]

33. Tamura, Y. 2003. Trends in antimicrobial agents for veterinary use and control measures of antimicrobial resistance. J. Jpn. Vet. Med. 56: 685-691.

34. Usui, M., Uchiyama, M., Iwanaka, M., Nagai, H., Yamamoto, Y. and Asai, T. 2009. Intracellular concentrations of enrofloxacin in quinolone-resistant Salmonella enterica subspecies enterica serovar Choleraesuis. Int. J. Antimicrob. Agents 34: 592-595. [Medline] [CrossRef]

35. Yamane, K., Wachino, J., Suzuki, S., Kimura, K., Shibata, N., Kato, H., Shibayama, K., Konda, T. and Arakawa, Y. 2007. New plasmid-mediated fluoroquinolone efflux pump, QepA, found in an Escherichia coli clinical isolate. Antimicrob. Agents Chemother. 51: 3354-3360. [Medline] [CrossRef]

36. Yang, H., Chen, S., White, D. G., Zhao, S., McDermott, P., Walker, R. and Meng, J. 2004. Characterization of multipleantimicrobial-resistant Escherichia coli isolates from diseased chickens and swine in China. J. Clin. Microbiol. 42: 3483-3489. [Medline] [CrossRef]

37. Yoshida, H., Bogaki, M., Nakamura, M., Yamanaka, L. M. and Nakamura, S. 1991. Quinolone resistance-determining region in the DNA gyrase gyrB gene of Escherichia coli. Antimicrob. Agents Chemother. 35: 1647-1650. [Medline] [CrossRef]

38. Xu, L., Ensor, V., Gossain, S., Nye, K. and Hawkey, P. 2005. Rapid and simple detection of $b l a_{\mathrm{CTX}-\mathrm{M}}$ genes by multiplex PCR assay. J. Med. Microbiol. 54: 1183-1187. [Medline] [CrossRef] 